\title{
MRI of the lung (2/3). Why ... when ... how?
}

\author{
J. Biederer • M. Beer • W. Hirsch • J. Wild • M. Fabel • \\ M. Puderbach • E. J. R. Van Beek
}

Received: 22 August 2011 /Revised: 20 November 2011 / Accepted: 19 December 2011 / Published online: 13 February 2012

(C) European Society of Radiology 2012

\begin{abstract}
Background Among the modalities for lung imaging, proton magnetic resonance imaging (MRI) has been the latest to be introduced into clinical practice. Its value to replace Xray and computed tomography (CT) when radiation exposure or iodinated contrast material is contra-indicated is well acknowledged: i.e. for paediatric patients and pregnant women or for scientific use. One of the reasons why MRI of the lung is still rarely used, except in a few centres, is the lack of consistent protocols customised to clinical needs.

Methods This article makes non-vendor-specific protocol suggestions for general use with state-of-the-art MRI scanners, based on the available literature and a consensus discussion within a panel of experts experienced in lung MRI. Results Various sequences have been successfully tested within scientific or clinical environments. MRI of the lung with appropriate combinations of these sequences comprises morphological and functional imaging aspects in a single
\end{abstract}

J. Biederer $(\bowtie) \cdot$ M. Fabel

University Hospital Schleswig-Holstein, Campus Kiel,

Department of Diagnostic Radiology,

Arnold-Heller-Straße 3, Haus 23,

24105 Kiel, Germany

e-mail: juergen.biederer@rad.uni-kiel.de

\section{Beer}

University Hospital Würzburg, Department of Radiology,

Josef-Schneider-Straße 2, Haus D31,

97080 Würzburg, Germany

\section{W. Hirsch}

University Hospital Leipzig, Department of Paediatric Radiology,

Liebigstrassse 20A,

04103 Leipzig, Germany

\section{J. Wild}

Academic Radiology, Royal Hallamshire Hospital Sheffield,

University of Sheffield,

Sheffield S10 2JF, UK examination. It serves in difficult clinical problems encountered in daily routine, such as assessment of the mediastinum and chest wall, and even might challenge molecular imaging techniques in the near future.

Conclusion This article helps new users to implement appropriate protocols on their own MRI platforms.

Main Messages

- MRI of the lung can be readily performed on state-of-theart 1.5-T MRI scanners.

- Protocol suggestions based on the available literature facilitate its use for routine

- MRI offers solutions for complicated thoracic masses with atelectasis and chest wall invasion.

- MRI is an option for paediatrics and science when CT is contra-indicated

Keywords Magnetic resonance imaging · Lung, sequence · Imaging protocol

M. Puderbach

Department of Radiology,

German Cancer Research Center (DKFZ),

Im Neuenheimer Feld 280,

69120 Heidelberg, Germany

M. Puderbach

Department of Diagnostic and Interventional Radiology,

Chest Clinics at University Hospital Heidelberg,

Amalienstrße 5,

69126 Heidelberg, Germany

\section{E. J. R. Van Beek}

Clinical Research Imaging Centre, University of Edinburgh,

47 Little France Crescent,

Edinburgh EH16 4TJ, UK 


\section{Introduction}

Increasingly, magnetic resonance imaging (MRI) of the lung is being advanced from a technological niche to the doorsteps of clinical routine imaging. The key technique for MRI of lung morphology is based on the resonant high-frequency signal of protons in tissues and liquids, so-called proton-MRI or ${ }^{1} \mathrm{H}$-MRI. The recent technical advances have helped MRI to challenge its well-known limitations as they are defined by the low proton density in the lung and the fast signal decay due to susceptibility artefacts at air-tissue interfaces [1]. Besides chest radiography, the most commonly employed first-line test for chest disorders, and computed tomography (CT), so far the most comprehensive and detailed modality for crosssectional and three-dimensional (3D) imaging of the lung, MRI is becoming an alternative, third method for the assessment of pulmonary diseases. Once broadly available and sufficiently robust, it will likely become a modality of choice for cases in which exposure to ionising radiation should be strictly avoided. This would comprise children, pregnant women and disorders requiring repeated examinations over prolonged periods, such as in patients with neutropenia, in which MRI could contribute significantly to lowering the cumulative radiation dose [2].

However, MRI offers additional advantages beyond the scope of X-ray-based techniques due to its functional imaging capacities. More than any other modality, MRI combines excellent soft tissue contrast and functional information [3]. It allows for multiple and repeated measurements and can be used to assess motion and perfusion of the thoracic organs. These advantages are already well appreciated in MRI of the heart, which combines morphological information (wall thickness, edema, scarring of the myocardium) with functional assessment (wall motion, contractility, ejection fraction) and viability/perfusion (e.g. at rest and during stress) [4]. Advances in MRI technology and a broad consensus on the basic imaging protocols have helped to establish a complex technology such as cardiac MRI within a relatively short period of time.

Since imaging technology has brought similar advances to MRI of the lung, it is basically the lack of comprehensive and standardised imaging protocols that delay its introduction for broader clinical use [5]. A number of applicable sequence techniques have been introduced and tested to various degrees. Every single sequence covers different aspects of lung pathology, e.g. liquid content of infiltrates or sharp delineation of solid nodules, etc. Sequence packages and customised protocols to combine strengths and diagnostic yields of the available sequences have been suggested (for an example of detailed vendor-specific recommendations see: Biederer J, Hintze C, Fabel M, Jakob PM, Horger W,
Graessner J, Bolster BD, Heller M (2011) MRI of the lungready ... get set ... go! Magnetom Flash 46:6-15; http://www. medical.siemens.com/siemens/en_GLOBAL/rg marcom_F BAs/files/apps/magazine/magnetom_flash/2011-05/Fla sh 46 einzel final.pdf). However, based on the platforms offered by different vendors, the protocols tend to be difficult to compare and make clinical introduction unnecessarily complicated. On the occasion of the first European School of Radiology courses on lung MRI and dedicated sessions at the European Congress of Radiology (ECR) 2010 and the European Society of Thoracic Imaging (ESTI) meeting 2010 as well as the meeting of the Chest Imaging Workshop (AG Thorax) at the German Roentgen Society (DRG), it was suggested that a group of experts in the field should generate a consensus statement on the current state of the art, indications and applications and future developments of lung MRI. After introducing the principles of the available technology in the first of this set of three articles [MRI of the lung (1/3)], it is the aim of the following lines to summarise the currently available protocols, comment on their clinical value based on the discussions within a panel of experts who are already practising lung MRI and to make updated uniform protocol suggestions for general use at state-of-the-art MRI scanners. A third manuscript will subsequently describe the current applications and future directions of lung MRI [MRI of the lung (3/3)].

\section{Expectancies to be met by MRI of the lung}

The prerequisites for the introduction of a new technique into the clinical setting are practicality of the application as well as robustness and reproducibility of image quality and diagnostic accuracy. The practicality of an MRI technique is further enhanced by a set of readily prepared protocols customised for specific clinical questions and available for standard scanners [1.5 Tesla (T)]. Preferably, additional features complicating the procedure such as the need for ECG-triggering or contrast material administration would be avoided. Furthermore, solutions for typical problems such as patients not being able to hold their breath or young children should be offered. In practice, it has been useful to design a common basic protocol trunk to cover the majority of expected clinical problems [1]. This basic protocol will be extended for specific questions such as the staging of malignancy, evaluation of pulmonary vessels and perfusion, etc. Furthermore, if the use of MRI is intended for emergency conditions, such as acute pulmonary embolism, fast and efficient procedures are required with in-room times that make it possible to squeeze the exam into a full MR schedule during the day [5]. Given the high investment into state-of-the-art MRI equipment and the running costs for maintenance and personnel, the key parameter for costeffectiveness of the method is in-room time. Based on the 
currently available standards, in-room times of $15 \mathrm{~min}$ for the basic protocol or an emergency examination were considered desirable [1]. Additional protocol extensions, e.g. contrastenhanced series, to detect tumour necrosis and pleural reaction/carcinosis or dynamic contrast-enhanced MRI and visualisation of respiratory motion contribute functional information should not exceed further 5-15 $\mathrm{min}$ in addition to the time needed for the basic protocol. To match this frame of expectancies as far as possible was the aim of the presented consensus on lung MRI protocol suggestions. Tables 1, 2, 3, 4 provide a systematic overview over indications for lung MRI and imaging protocol set-ups as well as a list of abbreviations/ glossary of terms in order to facilitate comparisons and crossvendor correlation throughout the manuscript.

\section{Experience with MRI for the evaluation of pathological conditions in the lung}

Pathological conditions in the lung related to changes of lung structure result either in an increase of lung density (collapse of lung with atelectasis, fluid accumulation inside the alveolar space and/or the lung interstitium, fluid and cell accumulation in infiltrates or growth of soft tissue resulting in nodules and masses) or decrease of lung density (overinflation due to air-trapping, enlargement of air spaces in emphysema) [6-10]. Given the well-known limitations of MRI in visualising low-proton-density structures with high susceptibility artefacts at tissue-air interfaces, any pathology resulting in an increase of lung density (and consequently

Table 1 Indications for lung MRI (in compliant patients)

\begin{tabular}{|c|c|c|}
\hline Role of MRI & Situation/pathological condition & i.v. contrast \\
\hline Situations that warrant the use of MRI & $\begin{array}{l}\text { Children } \\
\text { Pregnant women } \\
\text { Young patients with need for repeated imaging of the lungs } \\
\text { To replace CT if contrast media are contra-indicated } \\
\text { Scientific studies } \\
\text { Pharmaceutical trials } \\
\text { In discussion: lung cancer screening }\end{array}$ & $\begin{array}{l}\text { Optional } \\
\text { Optional } \\
\text { Optional } \\
\text { Optional } \\
\text { Optional } \\
\text { Optional } \\
\text { Optional }\end{array}$ \\
\hline $\begin{array}{l}\text { Indications covered by MRI } \\
\text { with advantages over CT }\end{array}$ & $\begin{array}{l}\text { Complicated thoracic mass (mediastinum and chest wall invasion) }{ }^{\mathrm{a}} \\
\text { Differentiation of atelectasis and pulmonary mass }{ }^{\mathrm{a}} \\
\text { Differentiation of mediastinal masses } \\
\text { Evaluation of respiratory mechanics } \\
\text { Diagnosis of pulmonary perfusion deficits (embolism, hypoxic vasoconstriction) } \\
\text { Cystic fibrosis (with perfusion study) }{ }^{\mathrm{a}}\end{array}$ & $\begin{array}{l}\text { Optional } \\
\text { Optional } \\
\text { Optional } \\
\text { No } \\
\text { Yes } \\
\text { Yes }\end{array}$ \\
\hline $\begin{array}{l}\text { Indications covered by MRI } \\
\text { as effectively as with CT } \\
\text { (if situation warrants use of MRI) }\end{array}$ & $\begin{array}{l}\text { Pneumonia } \\
\text { Atelectasis } \\
\text { Cystic fibrosis (without perfusion study) }{ }^{\mathrm{a}} \\
\text { Tuberculosis } \\
\text { Pulmonary nodules }(>3 \mathrm{~mm}) \\
\text { Sarcoidosis } \\
\text { Acute and chronic pulmonary embolism }{ }^{\mathrm{a}} \\
\text { Abnormalities of pulmonary venous drainage } \\
\text { Pulmonary arterial aneurysm } \\
\text { Lung sequestration } \\
\text { AV malformation (M. Osler) } \\
\text { Staging of lung cancer } \\
\text { Vasculitis (e.g. Wegener's) } \\
\text { Pleural effusion of unclear origin } \\
\text { Mesothelioma }\end{array}$ & $\begin{array}{l}\text { No } \\
\text { No } \\
\text { No } \\
\text { No } \\
\text { No } \\
\text { Optional } \\
\text { Yes } \\
\text { Yes } \\
\text { Yes } \\
\text { Yes } \\
\text { Yes } \\
\text { Yes } \\
\text { Yes } \\
\text { Yes } \\
\text { Yes }\end{array}$ \\
\hline $\begin{array}{l}\text { Indications covered less effectively } \\
\text { than with CT }\end{array}$ & $\begin{array}{l}\text { Pulmonary miconodules }(<3 \mathrm{~mm}) \\
\text { Interstitial lung disease }^{\mathrm{a}} \\
\text { Emphysema/COPD* }\end{array}$ & $\begin{array}{l}\text { Optional } \\
\text { Optional } \\
\text { Optional }\end{array}$ \\
\hline
\end{tabular}

${ }^{\text {a }}$ Specific topics covered with more detail in the third article of this series 


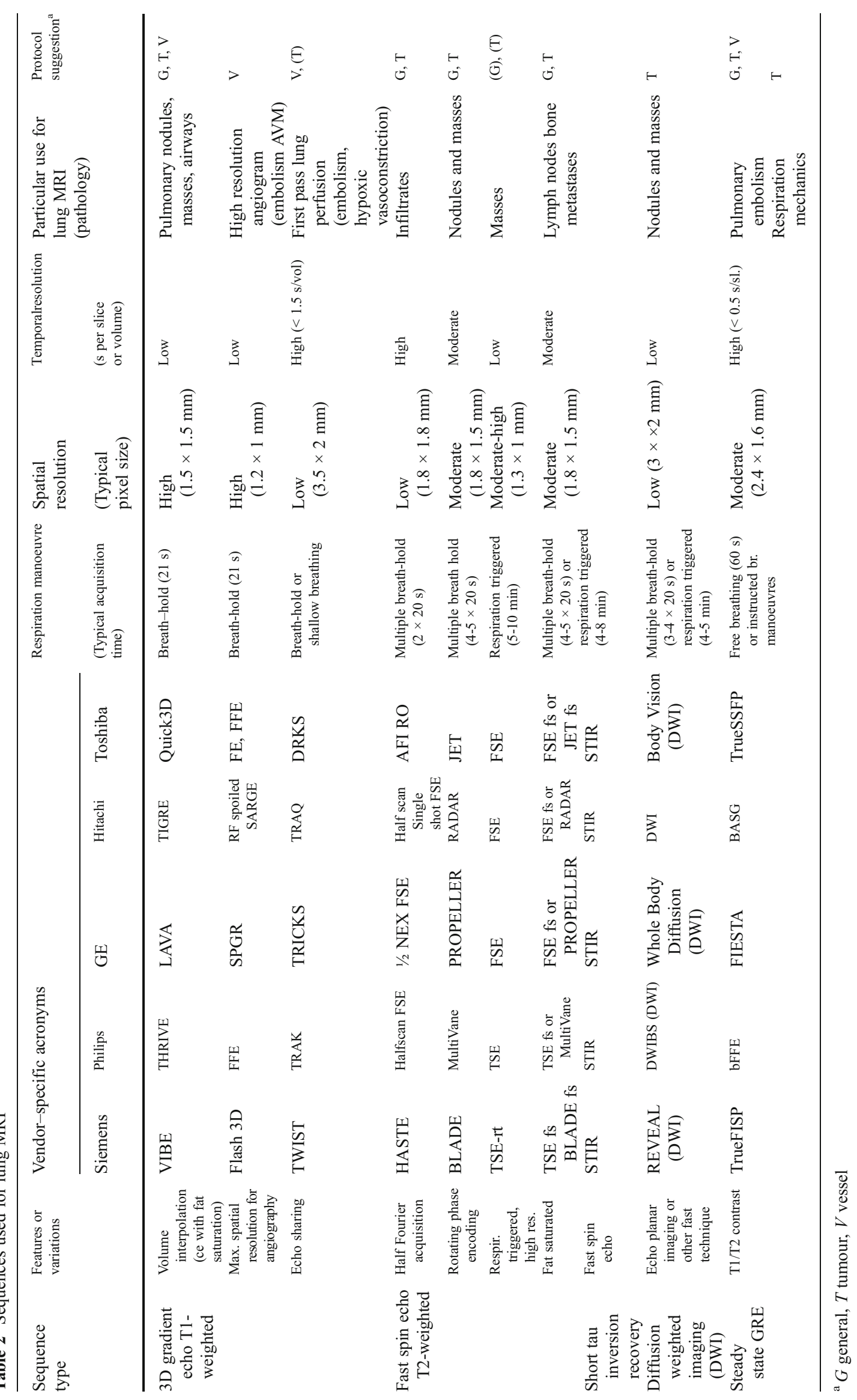




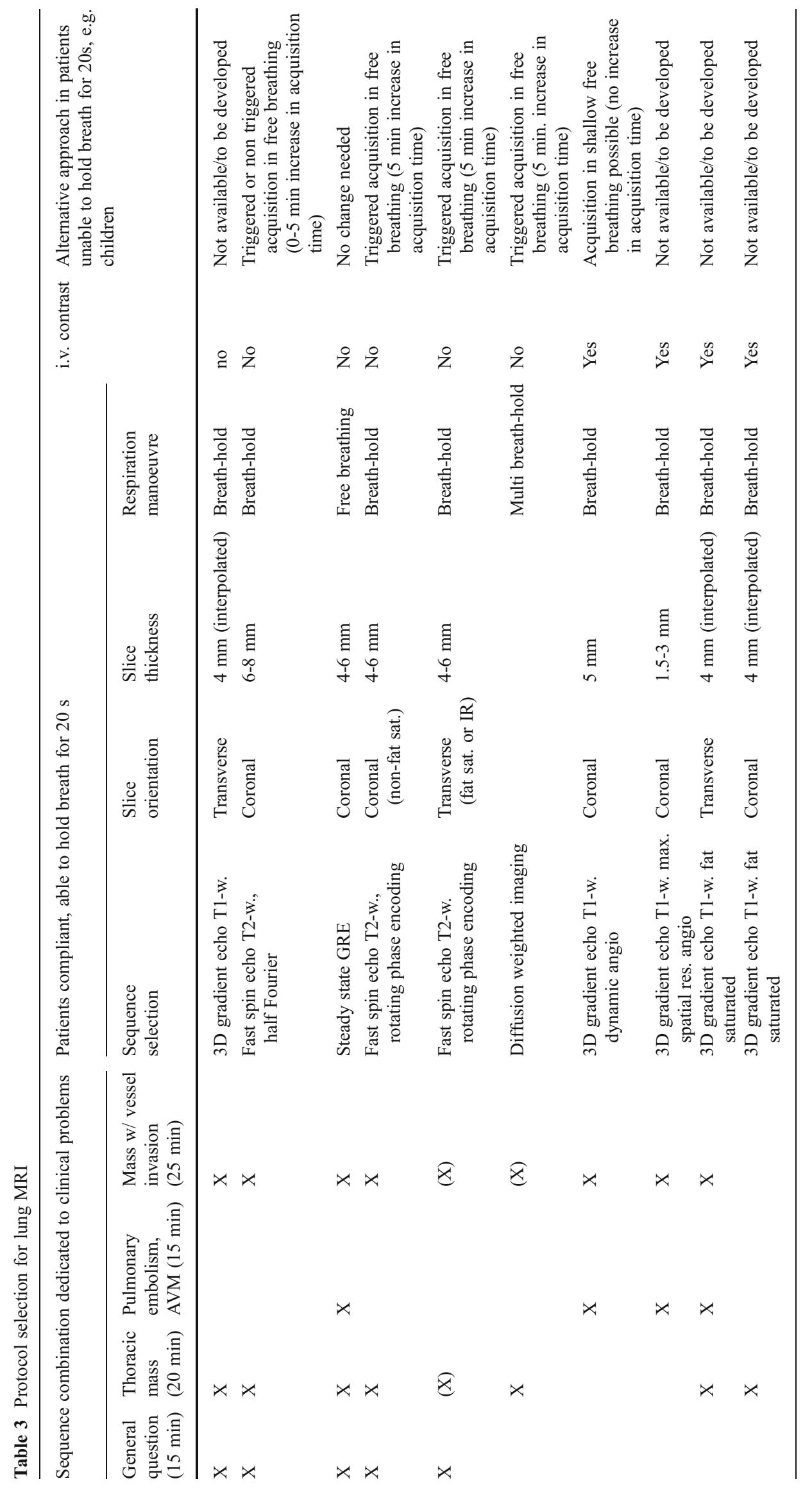


Table 4 List of abbreviations/acronyms as used in the text and in Tables 2 and 3

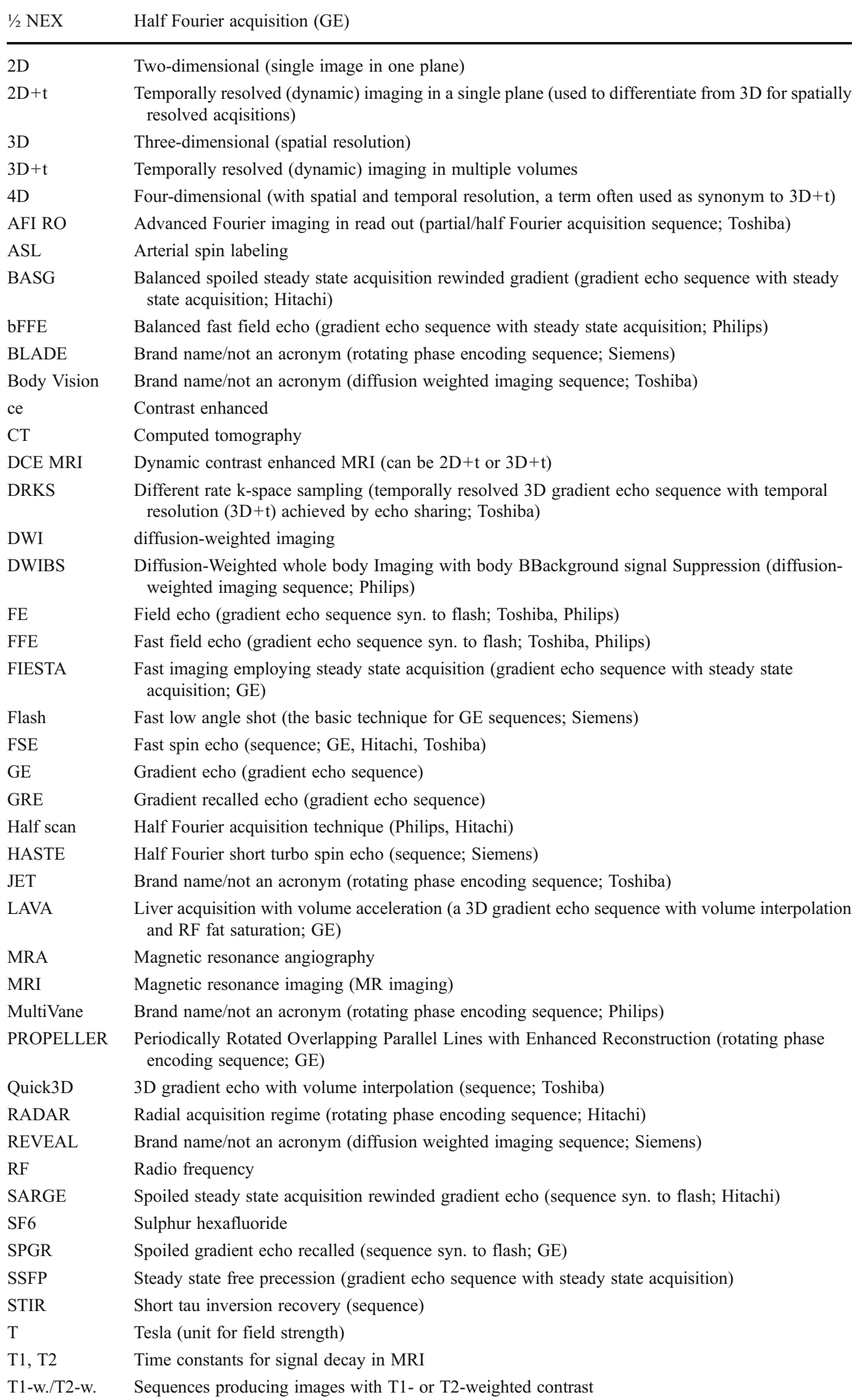


Table 4 (continued)

\begin{tabular}{|c|c|}
\hline $1 / 2 \mathrm{NEX}$ & Half Fourier acquisition (GE) \\
\hline THRIVE & $\begin{array}{l}\text { T1 high resolution isotropic volume excitation (a 3D gradient echo sequence with volume } \\
\text { interpolation and RF fat saturation; Philips) }\end{array}$ \\
\hline TIGRE & $\begin{array}{l}\text { Brand name/not an acronym (a 3D gradient echo sequence with volume interpolation and RF fat } \\
\text { saturation; Hitachi) }\end{array}$ \\
\hline TRAK & $\begin{array}{l}\text { Time-resolved angiography using keyhole (a temporally resolved 3D gradient echo sequence with } \\
\text { temporal resolution }(3 \mathrm{D}+\mathrm{t}) \text { achieved by echo sharing; Philips) }\end{array}$ \\
\hline TRICKS & $\begin{array}{l}\text { Time-resolved imaging of contrast kinetics (a temporally resolved 3D gradient echo sequence with } \\
\text { temporal resolution }(3 \mathrm{D}+\mathrm{t}) \text { achieved by echo sharing; GE) }\end{array}$ \\
\hline TrueFISP & $\begin{array}{l}\text { True fast imaging with steady state precession (gradient echo sequence with steady state acquisition; } \\
\text { Siemens) }\end{array}$ \\
\hline TrueSSFP & True steady state free precession (gradient echo sequence with steady state acquisition; Toshiba) \\
\hline TSE & Turbo spin echo (fast spin echo sequence; Siemens, Philips) \\
\hline TWIST & $\begin{array}{l}\text { Time-resolved angiography with stochastic trajectories (temporally resolved } 3 \mathrm{D} \text { gradient echo } \\
\text { sequence with temporal resolution }(3 \mathrm{D}+\mathrm{t}) \text { achieved by echo sharing; Siemens) }\end{array}$ \\
\hline VIBE & $\begin{array}{l}\text { Volumetric interpolated breath hold examination (a 3D gradient echo sequence with volume } \\
\text { interpolation and RF fat saturation; Siemens) }\end{array}$ \\
\hline
\end{tabular}

proton density) should be easy to detect, while a decrease of lung density will remain more challenging to visualise with MRI [8]. However, most conditions resulting in destruction and over-inflation of lung parenchyma, such as in emphysema, are associated with characteristic changes in the remaining lung parenchyma (fibrosis, septae, parenchymal distortion, bronchial wall thickening, etc.). Hence morphological MRI can still provide a correct diagnosis based on widened spaces of missing signal due to over-inflation and associated findings with increased signal intensity [11]. A direct visualisation of the airspace remains the domain of sophisticated imaging technology in specialised centres (see airways and lung ventilation disorders). Furthermore, changes in lung perfusion (hypoxic vasoconstriction, pulmonary embolism, congestion due to heart failure) result in changes of lung signal on MRI [7]. Non-enhanced MRI can directly visualise the vessel tree to detect macro-vascular pathology.

Pathological conditions with increased signal intensity

Quite a lot of experience exists for detection of pathological conditions that result in an increase of lung proton density. Experimental work has shown the high sensitivity of MRI with T2-weighted and proton-density weighted sequences for fluid accumulation inside the lung [12-14]. One can conclude that the sensitivity of MRI for pulmonary infiltrates is at least as good as with chest radiography and CT [2, 12, 15-17]. The majority of the applied protocols are based on T2-weighted or proton-density-weighted fast spin echo sequences, either with respiration gating or triggering or in breath-hold acquisition modes (Fig. 1).

Another key clinical demand is the detection of small solid or soft tissue lesions ("nodules"). The sensitivity of
MRI for lung nodules larger than $4 \mathrm{~mm}$ ranges between 80 and $90 \%$ and reaches $100 \%$ for lesions larger than $8 \mathrm{~mm}$ [18]. Depending on the sequence technique and the signal intensity of the lesions and given that conditions are optimal (i.e. patient can keep a breath-hold for $20 \mathrm{~s}$ or perfect gating/ triggering), a threshold size of 3-4 mm can be assumed for lung nodule detection with MRI [19, 20]. In comparison with CT, it might be even faster and more efficient to read lung MRI for pulmonary nodules, since they appear with bright signal against the dark background of the healthy lung tissue [21] (Fig. 2). Calcified nodules tend to disappear in the background, as they have no inherent signal, whereas contrast-filled vascular lesions will be highly visible on T1weighted images [22]. So far, a variety of sequence types has been evaluated for lung nodule detection with MRI. The spectrum comprises T2-weighted fast spin echo imaging with and without fat saturation [23-26], inversion recovery techniques [27], T1-spin echo [26, 28] and gradient echo sequences [29, 30].

The implications for protocol design are to include at least one T2-weighted or proton-density-weighted or short tau inversion recovery (STIR) sequence to cover infiltrates and nodular lesions with high fluid content. This should be combined with a second sequence to cover nodules with high signal on T1-weighted images as well. In particular, for the detection of malignant lesions with hyperperfusion and intense enhancement, the i.v. administration of paramagnetic contrast material might be helpful and even increase detection rates. However, this has not yet been confirmed by appropriate studies so far.

Not yet clarified is the role of diffusion-weighted imaging (DWI) of lung lesions. DWI is recommended for the wholebody staging of lung cancer, including mediastinal 
Fig. 1 A 66-year-old male patient with fever and coughing, clinically suspected pneumonia. The plain chest X-ray (a) demonstrates a dense infiltrate in the left lower lung lobe which is confirmed on non-contrastenhanced low dose CT (b; arrows). The patient volunteered to undergo MRI on the same day. Multi-breath-hold coronal T2-weighted fast spin echo (c) and single breath-hold T1-weighted 3D GRE imaging (d) as well as free breathing coronal steady state SSFP (e) and multi-breath-hold fat-saturated T2-weighted fast spin echo series (f) clearly demonstrate the infiltrates with particularly high signal on T2-weighted images
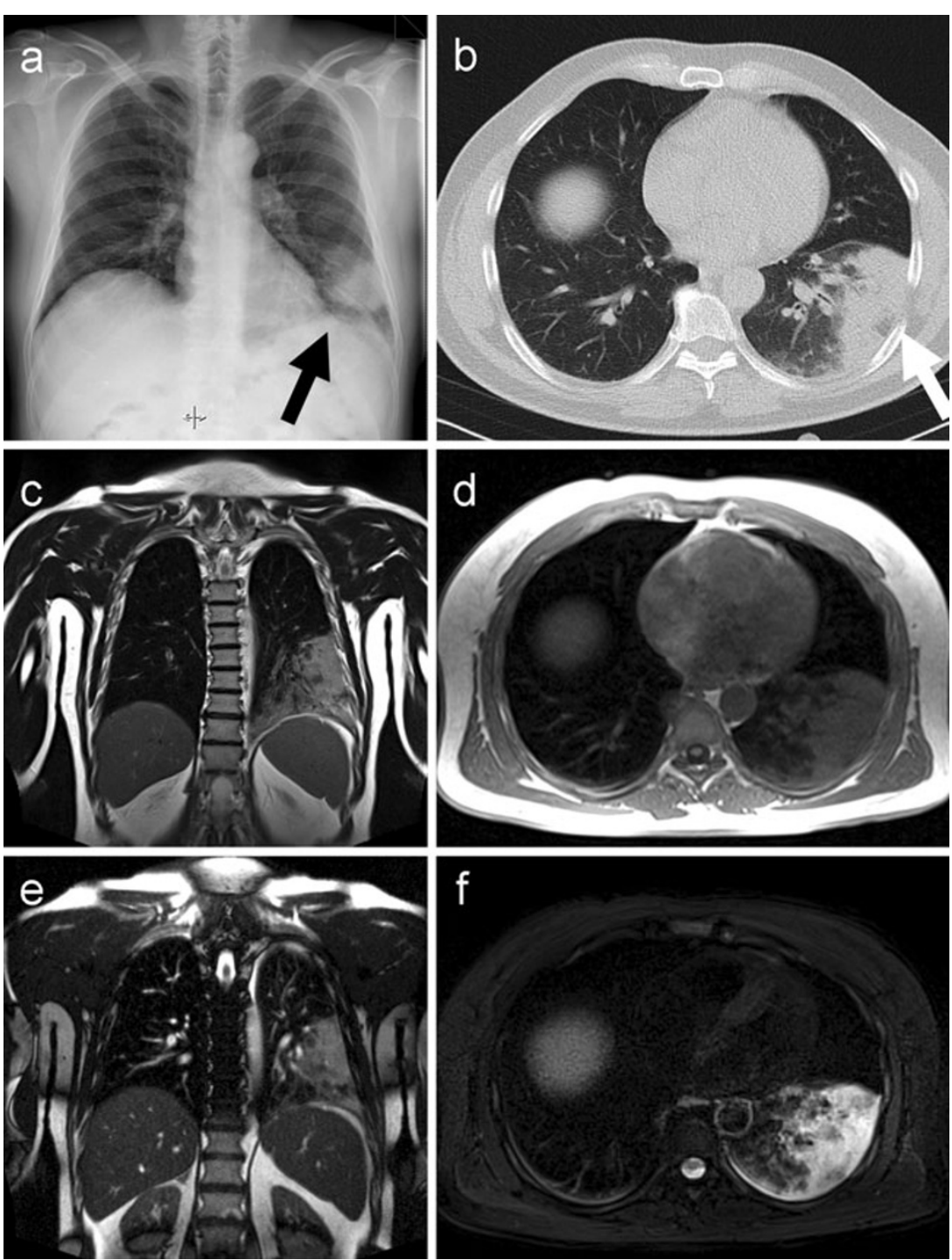

metastases [31, 32]; however, in the chest a clear advantage of DWI over other MRI protocols has not been confirmed so far $[33,34]$. STIR sequences might be even more sensitive for the detection and classification of lung cancer and mediastinal metastases than DWI [35-37]. One potential role of DWI might be to predict tumour invasiveness for clinical stage IA non-small-cell lung cancer and to separate the mass from atelectasis [38, 39]. The role of DWI sequences for the differentiation of malignant or benign lung lesions or for the discrimination of subtypes of lung cancer remains controversial [37, 40-42]. In the authors' own experience, DWI helps to demarcate lesions adjacent to the pleura (Fig. 3), assess mediastinal extension and may serve as a "second reader" for detection of small nodules. Thus, it makes sense
Fig. 2 A 64-year-old woman with the incidental finding of an unspecific, 4-mm nodule in the right middle lobe. The nodule (open arrow) is clearly depicted on the coronal multi-breath-hold T2-weighted (a) and transverse contrast-enhanced, fat-saturated 3D GRE images (b)
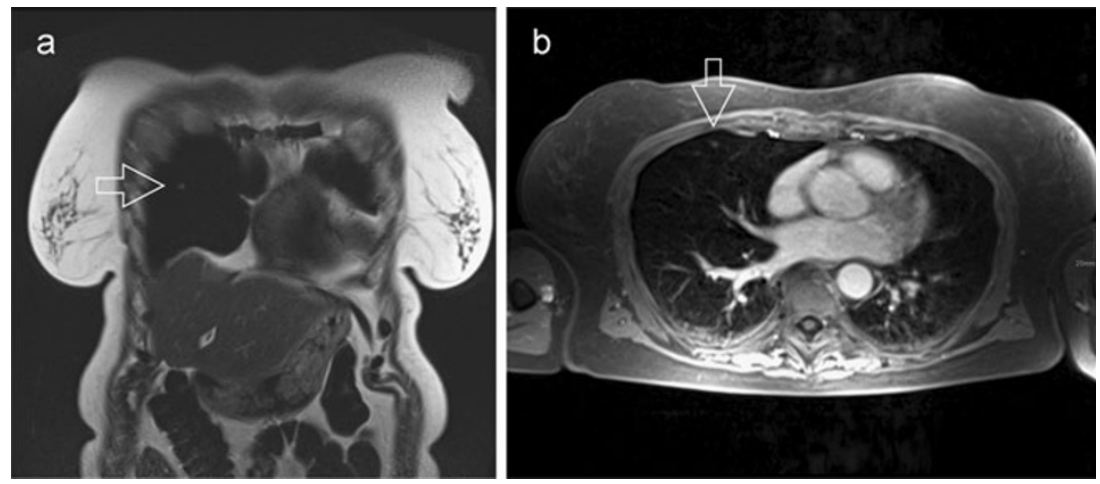
Fig. 3 Diffusion-weighted imaging highlighting pleural metastases of hepatocellular carcinoma at the right diaphragm (left, open arrow). The same spots are only hardly visible on the post-contrast fatsaturated breath-hold T1weighted 3D GRE sequence (right).
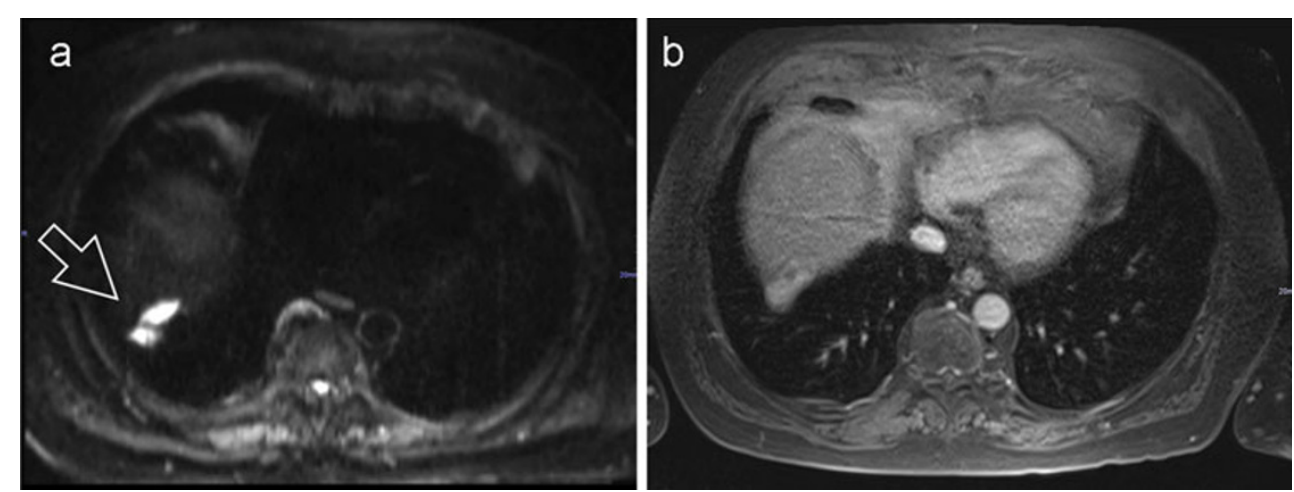

to include a fast DWI acquisition in the protocol recommendations for MRI of the lung [43, 44].

Vessels and lung perfusion disorders

Thus far, approaches for disorders of pulmonary perfusion have been aimed at either the visualisation of the pulmonary arterial tree (MR angiography) or at the assessment of lung parenchyma signal changes associated with perfusion on contrast-enhanced or non-contrast-enhanced scans.

MR angiography and MR perfusion have mainly been applied for the study of acute pulmonary embolism, a key clinical entity to demonstrate diagnostic accuracy. Many approaches include direct visualisation of the thrombus inside the pulmonary artery either with positive or negative contrast against the signal of the flowing blood. Presently, fast steady state free precession gradient echo sequences (SSFP-GRE) appear to be one of the most effective techniques. They display the lung vessels with bright signal, against which thrombotic material is contrasted with low signal intensity. A sensitivity of $90 \%$ and a specificity of $97 \%$ have been reported for acute segmental pulmonary embolism [45-47]. Another approach has used double inversion recovery, demonstrating stagnant blood clot as bright signal, but insufficient data exist as to the diagnostic accuracy at this time [48, 49].

The presently most powerful technique, however, appears to be high spatial resolution MR angiography with T1-weighted contrast-enhanced 3D gradient echo acquisitions with k-space centred contrast bolus in a breath-hold [50]. For optimum contrast, it is recommended to use a power injector and selected injection protocols [51]. Several studies have shown very encouraging results for MR angiography (MRA) in the workup of patients with suspected pulmonary embolism [52-55]. Most recently, the PIOPED III study involving seven centres and 371 patients with suspected pulmonary embolism demonstrated sufficient image quality in only $75 \%$ of the patients. Sensitivity and specificity for acute pulmonary embolism were $78 \%$ and $99 \%$, respectively [56]. The most frequent reason for insufficient image quality was dyspnoea, coughing or insufficient timing of contrast material injection. To overcome the problem of bolus timing, other approaches favoured timeresolved MRA with multiple acquisitions of lung volumes using very fast 3D gradient echo techniques. Extremely short acquisition times of $1.5 \mathrm{~s}$ or less were achieved by combining parallel imaging and data sharing [57]. With a so-called 4D MRA or dynamic first-pass perfusion MRI [also known as dynamic contrast enhanced (DCE)-MRI], Ersoy et al. [58] realised a sensitivity of $98 \%$ for lobar and $92 \%$ for segmental embolism.

The development of dynamic first pass perfusion MRI has opened further perspectives in studying lung parenchyma perfusion in other disease conditions than pulmonary embolism as well. Limited to one plane, the temporally resolved so-called two-dimensional dynamic $(2 \mathrm{D}+\mathrm{t})$ perfusion MRI reaches excellent temporal resolution of up to 10 images/s with reasonable spatial resolution [59]. However, volume coverage needs multiple series and contrast injections, which are usually not feasible. Thus, 4D approaches are usually preferred. Other techniques such as arterial spin labeling (ASL) based on intrinsic contrast of magnetised, inflowing blood into the imaging plane or volume have been investigated and applied for scientific studies, but have not yet found their way into clinical applications [60, 61].

The visual evaluation of 4D MRI image sets is facilitated by subtraction of the non-enhanced from the contrastenhanced image signal, which results in a bright display of the contrast-enhanced lung vessels and parenchyma. The clinical value of lung perfusion studies with visual and semi-quantitative evaluation is being tested in the assessment of lung perfusion deficits in cystic fibrosis patients who suffer from mucus retention and hypoxic vasoconstriction (Fig. 4) [62-64]. The perfusion series allows indirect visualisation of lung parenchyma abnormalities due to emphysema or conditions such as pneumothorax due to the absence of perfused lung tissue.

Airways and lung ventilation disorders

Direct visualisation of the airways with MRI is limited to airways in excess of $3 \mathrm{~mm}$ diameter, unless filled with bright materials such as retained mucus in cystic fibrosis patients 

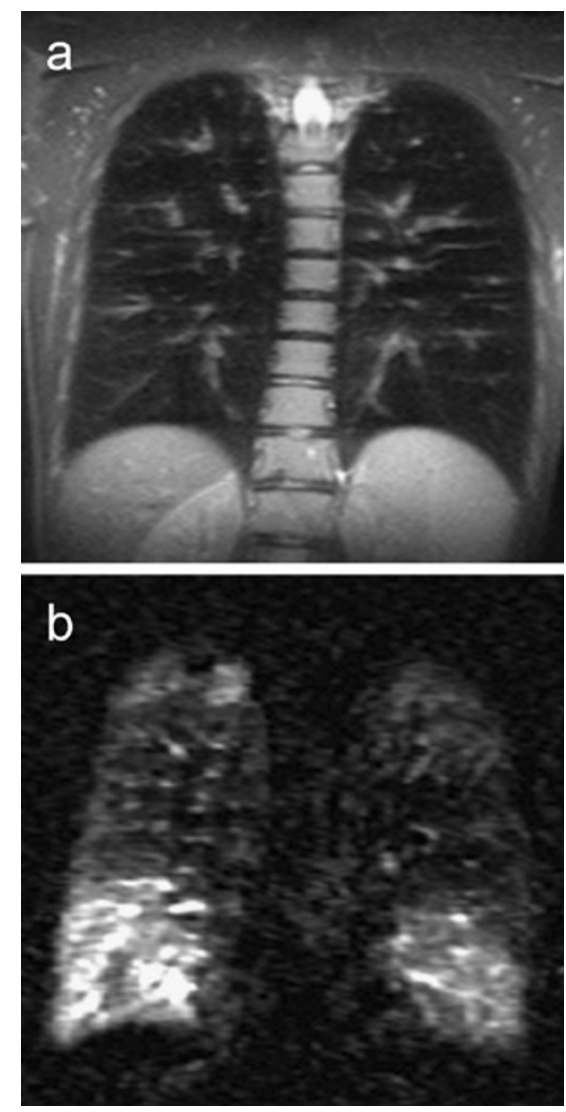

Fig. 4 An 18-year-old male cystic fibrosis patient, coronal T2weighted half Fourier fast spin echo sequence (a) and coronal subtraction perfusion image (b). Notice the severe mucus plugging in the morphological T2-weighted image. The subtraction perfusion image shows correspoding areas with perfusion loss due to hypoxic vasoconstriction. Due to redistribution of perfusion both lower lobes show a high perfusion signal

[65, 66] (Fig. 5). In young healthy subjects, lung MRI depicted airways down to the first subsegmental level; however, in parts of the lung subject to cardiac pulsation, detection rates were significantly lower [67]. As expected from the higher spatial resolution, high resolution CT is still superior to MRI in the depiction of small peripheral airways.

However, MRI is capable of studying dynamic processes, e.g. to analyse respiratory motion of the lung, diaphragm and relationships of tumours to chest wall boundaries. The technical advances described above for $2 \mathrm{D}+\mathrm{t}$ MRI or $3 \mathrm{D}+\mathrm{t}$ MRI with different variants of T1-weighted gradient echo or steady state free precession sequences have been applied for this purpose $[68,69]$. One of the most important clinical applications will be radiotherapy planning for organs with respiration-correlated motion $[70,71]$. Lung ventilation can be estimated from regional volume changes [72].

Since air has no signal on MRI, ventilation can only be visualised directly with hyperpolarised noble gases [73], sulphur hexafluoride (SF6) [74] or tracked indirectly by signal changes after inhalation of $100 \%$ oxygen [75] or
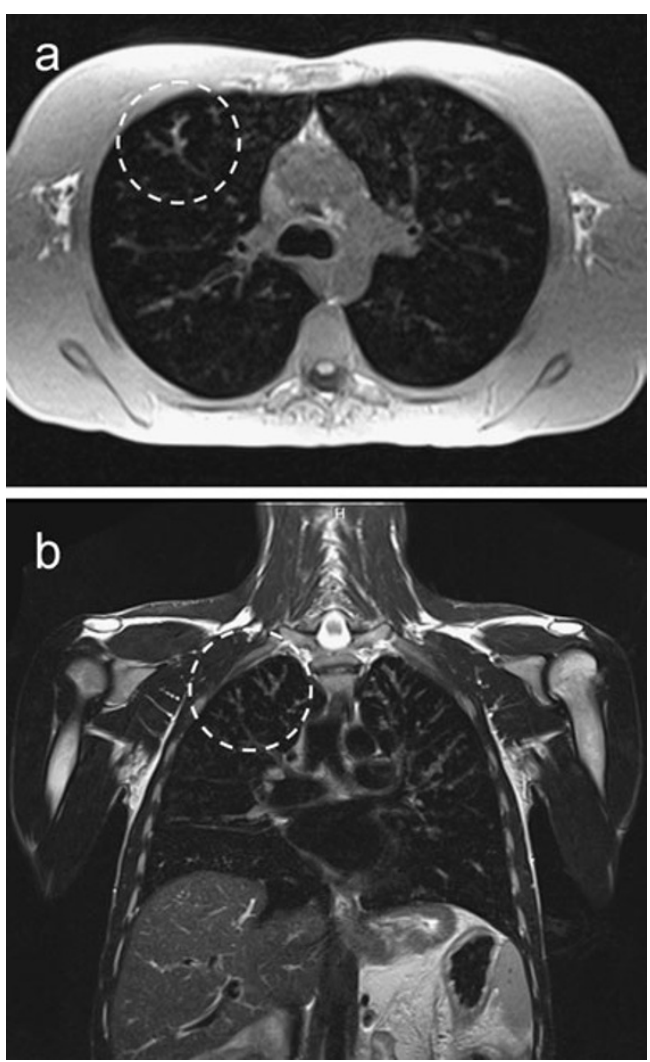

Fig. 5 Lung MRI of a 37-year-old male patient with cystic fibrosis. Coronal T2-weighted, respiration triggered and transverse breath-hold T1-weighted 3D GRE images show peripheral airways with enhanced signal due to mucus plugging. Note the "tree-in-bud" sign similar to the typical appearance on CT (dashed circles)

inhalation of aerosol of a paramagnetic contrast agent [76]. Despite the fascinating scientific publications obtained with these approaches, most of them are still remote from clinical application. Presently, the use of dynamic image acquisitions in single $2 \mathrm{D}$ planes $(2 \mathrm{D}+\mathrm{t})$ to study respiratory motion appears to be closest to routine use.

\section{Further aspects to be covered}

Apart from the conditions mentioned above, the spectrum of findings to be covered comprises a wide range of disorders, ranging from pleural pathology (effusion, empyema, tumour with chest wall infiltration, pneumothorax) to mediastinal involvement (masses, lymphnodes, vascular pathologies) and the assessment of the adjacent upper abdomen (e.g. adrenal and liver metastases in lung cancer). It would be beyond the scope of this article to discuss the role of MRI in all of these conditions, since this is well covered by standard textbooks. Nevertheless, any suggested standard protocol for lung diseases should offer sufficient coverage of the surrounding structures, which may then be further examined with dedicated protocols. A wide field, for instance, is the interaction between the heart and the lung. To keep within 
the frame of this article, the possible useful combinations of lung MRI protocol components with heart MRI protocols will not be discussed explicitly. However, their clinical value is obvious and building flexibility into the protocol trees is suggested, which would allow the user to combine protocols as required.

\section{Getting started (or what you may need to know to avoid disappointments)}

More than in X-ray or CT, image quality in MRI depends on patient compliance. As discussed above, it can be expected that some sequences may produce unsatisfactory results in individual patients. Therefore, the use of fast imaging protocols is advocated, with additional sequences to allow for with a certain degree of redundancy to compensate for any failed acquisitions [1]. Excellent results will be obtained in young, compliant subjects with good breath-hold capability. In contrast to this, it may become most difficult to obtain diagnostic image quality in non-compliant, obese patients unable to hold their breath or unable to understand and follow the breathing commands. For the latter, parts of the protocol can be acquired in free breathing, either due to their fast acquisition scheme or with respiration gated or triggered acquisition modes. Respiration-triggered versions of the T2-weighted fast spin echo sequences are available for uncooperative patients who have difficulties with breath-holding (their application increases the total in-room time by $10 \mathrm{~min}$ ). Technicians will test patient compliance early on in the study, and can adjust the sequences accordingly. However, it needs to be emphasised that coaching of patients is very important to gain most information from any MRI scan. Respiration belts may be helpful, since this allows for monitoring patient compliance even during image acquisition [1].

As discussed in the previous article of this series in detail [MRI of the lung (1/3)], the following protocol suggestions can be transferred from 1.5- to 3-T MR scanners [17]. While most sequences, in particular 3D gradient echo techniques, profit from a higher lesion-to-background contrast to a certain degree, artefacts may deteriorate the image quality of steady state gradient echo sequences [17, 21, 77]. Overall, the changes of image quality with transfer of the concept to $3 \mathrm{~T}$ are acceptable or even positive for most sequence types.

Imaging paediatric patients

The lack of radiation exposure makes MRI of the lung particularly attractive for paediatric radiology. Depending on experience with the technology and the availability of scanner time, MRI has become the first-line cross-sectional imaging technique for pulmonary disease in many departments [78]. The available sequence techniques are the same as for adult patients, but characteristic features of the paediatric setting influence the choice of protocols. First of all, breath-hold imaging is frequently not possible in young children up to 8 years. Depending on the size of the patients and their ability to comply with the procedure and breathing instructions (sometimes even 6-year-old or even younger children are capable of holding their breath on command), it appears useful to prepare a separate protocol tree not only with motion compensated protocols but also with adjusted fields of view, slice thickness and in-plane resolution with optimised signal-to-noise ratios for smaller subjects [79].

Two basic strategies have been pursued for motion compensation: fast single shot imaging with very short acquisition time and respiratory gating/triggering of fast spin echo techniques. For the first approach, steady state (SSFP) or partial Fourier single shot sequences (e.g. HASTE) have been successfully implemented [80]. SSFP sequences allow for a rapid acquisition of ten slices with breath-hold times below $10 \mathrm{~s}$, even on low-field MRI systems. Alternatively, they can be performed during free breathing [81, 82]. Typically, these fast and robust sequences would be used for an
Fig. 6 A 6-year-old child with lung metastases of osteosarcoma. Both acquisitions, the free breathing steady state free precession series (a) and the respiration triggered (navigator triggered) series (b) show a large mass with high signal intensity in the right upper lung lobe in expiration
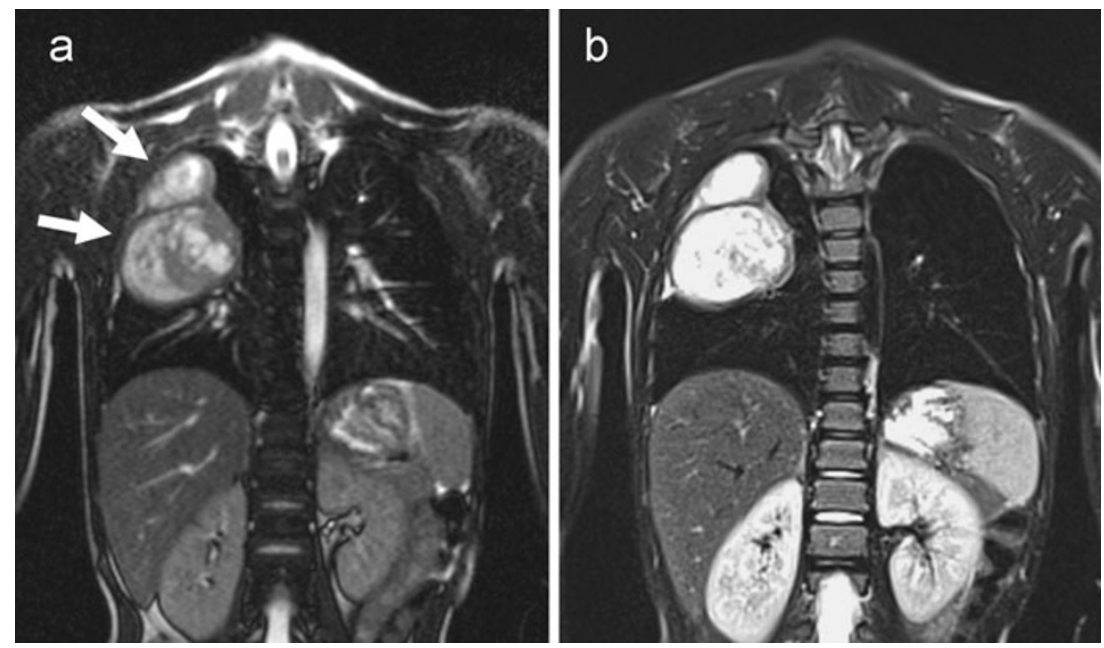

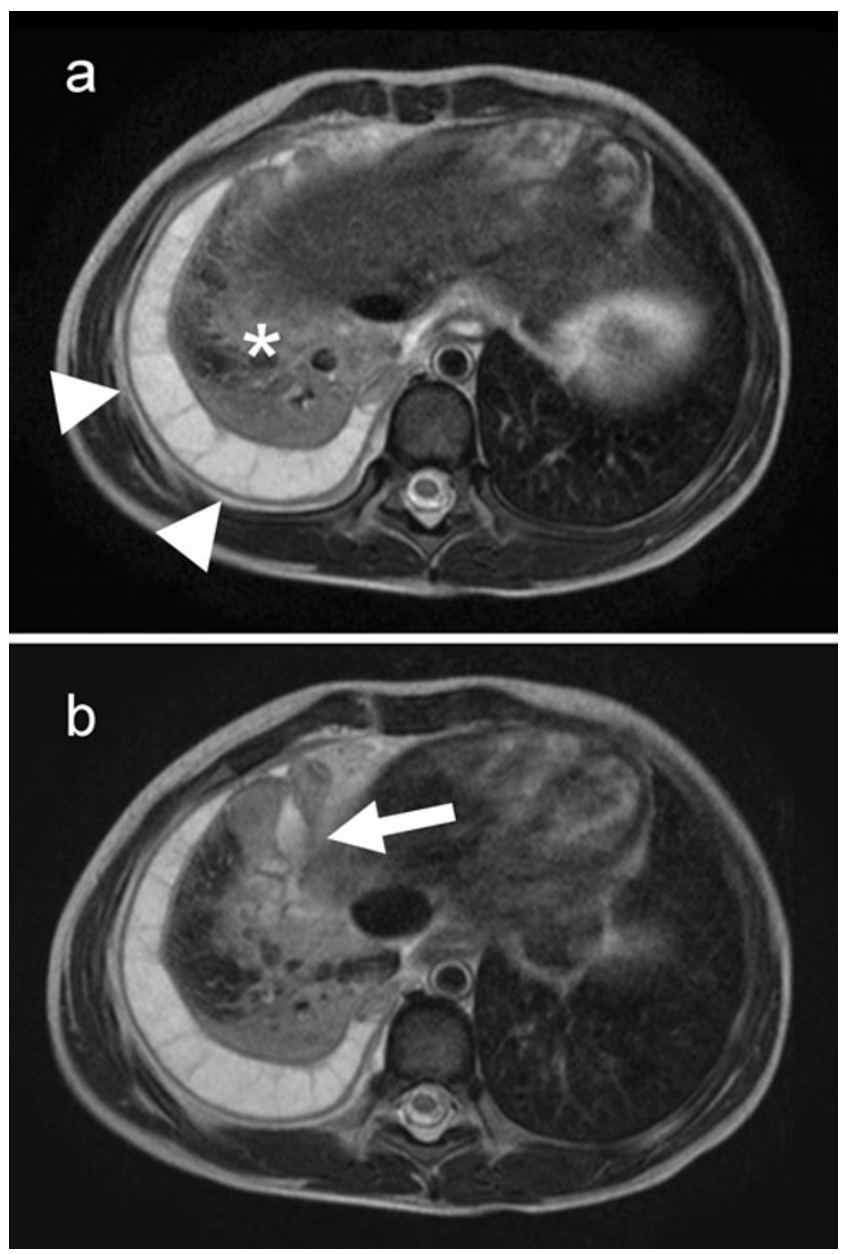

Fig. 7 Pneumonia (asterisk), chambered pleural effusion (arrowheads) and abscess (arrow) in the right lower chest of a 6-year-old child, images acquired in T2-weighted triggered fast spin echo technique

initial overview with further high-resolution acquisitions in the second part of the examination. The second approach, gated or triggered acquisition, increases imaging time but provides better spatial resolution and soft tissue contrast (Figs. 6, 7) $[79,83]$. The higher respiration frequencies of young children are of a certain advantage since they help speeding up the acquisition. In most cases, the difference to non-gated acquisitions is much less than in adult subjects with low respiration rates. T2-weighted fast spin echo sequences can be applied with repetition times of $2000 \mathrm{~ms}$ or less, usually triggered to the expiratory phase, which is around $2 \mathrm{~s}$, depending on the individual respiration frequency. This time frame allows for excellent T2-weighted images without relevant motion artefacts [84]. Depending on the available hardware and specific experience of the team, both mechanical (respiratory belt or cushion technique [84]) or image-based (e.g. navigator) devices for the detection of respiratory motion can be applied with good results. A radial read-out scheme of the $\mathrm{k}$-space further improves the robustness against motion artefacts. The application of additional cardiac triggering may be helpful in specific cases, but paid for with a significant increase of acquisition time [85].

A second characteristic feature of the paediatric setting is the need for sedation or general anaesthesia in many cases. Sedation will normally be avoided as much as possible, but may be indispensable. In low-field lung MRI it remains a matter of debate (Rupprecht et al. [82] versus Sanborn et al. [86]). For propofol sedation, an incidence of up to $42 \%$ of dorsal atelectasis has been described [87]. Other groups use chloral hydrate or phenobarbital and report less frequent atelectases (personal communication). However, it is important to know this condition, since it may mask relevant pathology. In case of doubt, additional scans in the prone position may be warranted. In case exact correlation with $\mathrm{CT}$ is needed, e.g. when switching follow-up examinations from CT to MRI, it may be useful to acquire the MRI examination with elevated arms. However, this position is usually not tolerated by children for more than $15-20 \mathrm{~min}$ and can not be generally recommended.

\section{Protocol suggestions}

Based on the published experience and matching the abovestated expectancies, Table 1 summarises a selection of
Fig. 8 Recent fracture of the left 5 th rib as incidental finding in a 29-year-old female volunteer with left chest pain, hardly visible on the non-contrast enhanced T1-weighteg breath-hold 3D GRE series (a) but with bright signal on the T2-weighted fat saturated image from an multiple breath-hold series (b, arrow)
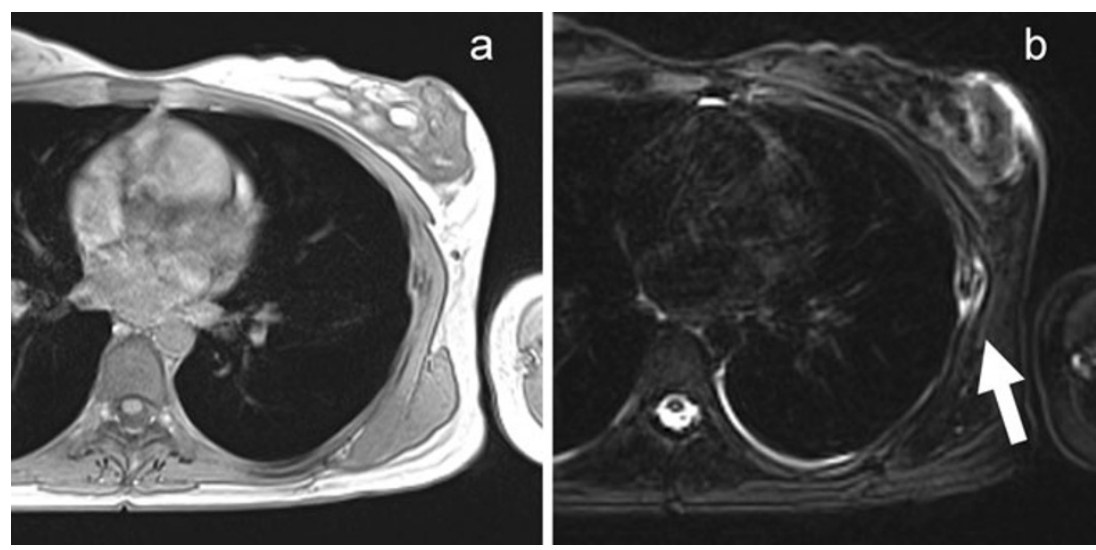


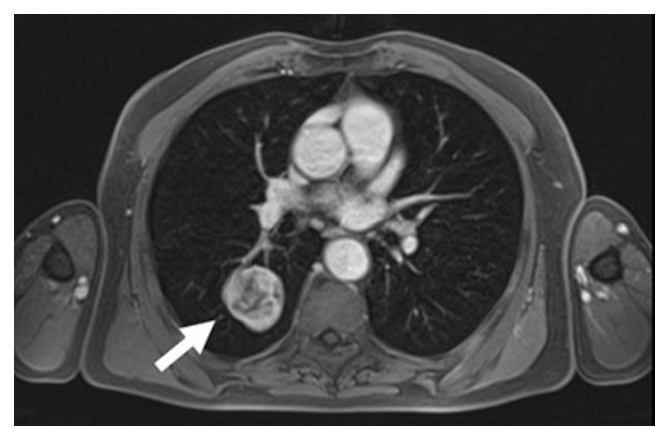

Fig. 9 A 77-year-old male patient with adenocarcinoma in segment 6 of the right lower lung lobe (arrow; transverse contrast-enhanced breath-hold 3D GRE study)

available sequences and their potential diagnostic yield for lung MRI. For the overview of concept, details on sequence technology and parameters are left out here, since dedicated protocols are described in the literature and may vary in between different vendors [88-91]. Adjusted to the size of the patient, the fields of view (FOVs) would be typically $450-500 \mathrm{~mm}$ in coronal and approximately $400 \mathrm{~mm}$ in transverse acquisitions with matrices of 256-384 pixels (for triggered fast spin echo series up to 512) resulting in pixel sizes smaller than $1.8 \times 1.8 \mathrm{~mm}$. Slice thicknesses for the $2 \mathrm{D}$ acquisitions would range from 4 to $6 \mathrm{~mm}$. Three-dimensional acquisitions for imaging lung morphology in transverse orientation would use slice thicknesses of $4 \mathrm{~mm}$ or less, pulmonary angiography in coronal orientation $2 \mathrm{~mm}$ or less [88]. This is in line with the QA guidelines for thoracic MRI published by the German Board of Medicine [92].

A basic or general study combines T1-weighted and T2weighted images based on gradient echo and fast spin echo sequences, respectively [90]. T1-GRE sequences are available as $3 \mathrm{D}$ acquisitions and may, therefore, be limited to a volume acquisition of the chest in one breath-hold. Fast T2- weighted spin echo sequences should cover at least two planes, e.g. with a half Fourier breath-hold acquisition in coronal and a transverse orientation. This results in a high sensitivity of the protocol for infiltrates and small nodular lesions. To improve the sensitivity of the protocol for mediastinal lymph nodes and bone lesions (e.g. metastases), a STIR or fat saturated T2-fast spin echo sequence should be added. Since rib metastases are easier to detect on transverse slices, this orientation should be preferred (Fig. 8). The list of "must" sequences (in the table indicated by $G$ for "general protocol") is concluded with a coronal steady-state free precession sequence in free breathing, which contributes to a high sensitivity for central pulmonary embolism and gross cardiac or respiratory dysfunction. As an option (but at the cost of additional imaging time exceeding the intended limitation to $15 \mathrm{~min}$ ), motion-compensated T2-weighted fast spin echo sequences can be added to improve the depiction of masses with chest-wall invasion $[1,5]$.

With this selection of non-contrast-enhanced sequences, the study covers some of the most common potential findings, including pneumonia, atelectasis, pulmonary nodules or masses, mediastinal masses (lymphoma, goiter, cyst, thymoma) and acute pulmonary embolism [5]. Depending on the initial findings following the basic protocol, additional contrast-enhanced acquisitions may be required, which would use the same type of volume interpolated 3D-GRE sequence, but now with fat saturation to improve the visibility of contrast-enhanced tissues and mediastinal lymph nodes ( $T$ "tumour protocol"). Although the 3D sequences cover the whole chest, in-plane resolution is optimised in either transverse or coronal section (Fig. 9). Since acquisition times are just one breath-hold, it appears to be feasible to acquire the 3D GRE studies in at least transverse and coronal planes. In particular for the staging of lung cancer, it might be helpful for the detection of small lymph nodes

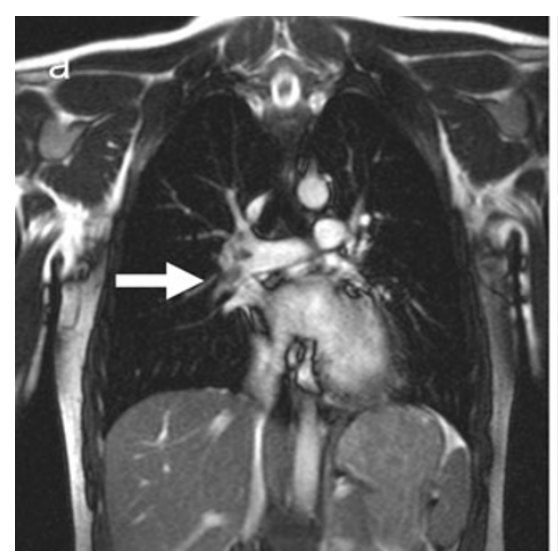

Fig. 10 An 18-year-old female patient with clinical suspicion (dyspnoea and elevated D-dimers) of acute pulmonary embolism. The steady state free precession study shows an embolus inside the right pulmonary artery (a, arrow) that is also clearly depicted in the
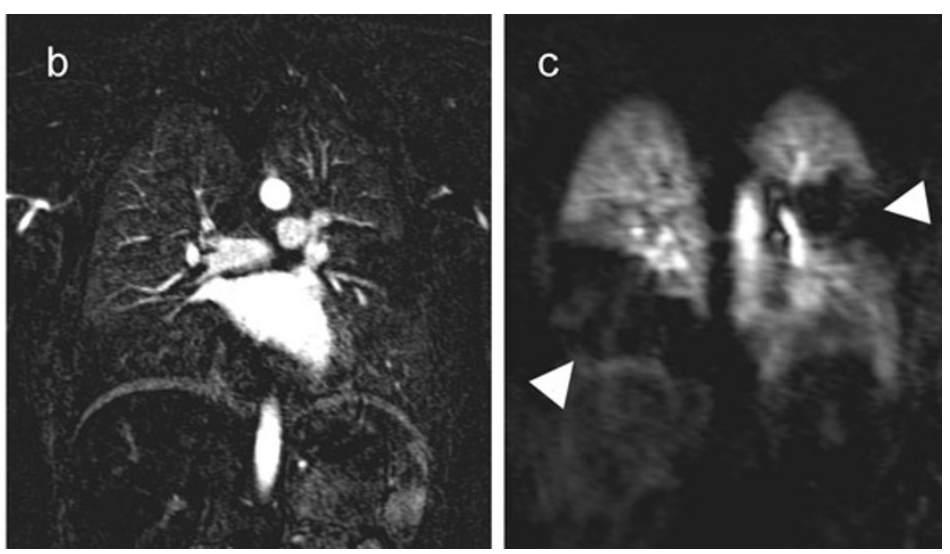

subtraction images form the contrast enhanced 3D flash MRA (b). The subtraction of the first pass perfusion study (c) confirm large perfusion deficits in the right lower lobe and a posterior segment of the left upper lobe (arrowheads) 
metastases to add a diffusion-weighted sequence as well. Both sequences extend the total imaging time by approximately 5 min [1].

The available options for imaging disorders of lung vasculature comprise three components: First of all, the free breathing steady state gradient echo (SSFP-GRE) study which is also part of the general protocol. Then two variations of 3D gradient echo based contrast enhanced MRA: (1) a time-resolved, low spatial resolution acquisition for first pass perfusion imaging and (2) a high spatial resolution acquisition for a breath-hold angiogram (Fig. 10). Depending on the performance of the MR scanner, the dynamic study produces a comprehensive lung perfusion study with excellent temporal resolution and at the same time serves for the determination of optimum contrast bolus timing for the acquisition of the high resolution angiogram. The "vessel protocol" ( $V$ in Table 1$)$ optimises the sensitivity for acute and chronic pulmonary embolism, arterio-venous (AV) malformation (e.g. Osler's disease), lung sequestration, pulmonary arterial aneurysm, abnormalities of pulmonary venous drainage and any other pathology of lung vasculature [5]. As in other parts of the protocol recommendations, it is suggested to combine different fast imaging sequences to increase sensitivity and specificity of the examination [93]. Therefore, Kluge et al. [94] suggested combinations of different available MRI techniques for the detection of pulmonary embolism [5]. The lung vessel imaging branch of the protocol tree can be used just for a study of vascular pathology, e.g. in suspected acute pulmonary embolism or in combination with the tumour protocol for the comprehensive evaluation of a central mass with vessel invasion.

These protocol suggestions cover all expected clinical questions with different branches of the protocol tree for specific problems. They are a good point to start from and allow users to make up their own branches based on the suggested components.

\section{Conclusions}

With a comprehensive protocol approach as proposed, MRI of the lung can be readily performed on most state-of-the-art 1.5-T MRI scanners. Breath-hold imaging or triggered acquisitions, together with advanced sequence technology (e.g. parallel imaging and rotating phase encoding), make image quality reasonably robust against artefacts induced by respiratory motion and heart action. Parts of the protocol produce images of diagnostic quality even in uncooperative patients. This allows for appreciation of the specific features of lung MRI related to its excellent soft tissue contrast and functional imaging capacities. The sensitivity of these protocols for infiltrates is at least similar to X-ray and CT. In lung nodule detection, MRI is superior to X-ray and almost as sensitive as CT. A dedicated selection of sequences for imaging lung vasculature allows for the detection of pulmonary embolism with high sensitivity and specificity. In respect to lung perfusion and respiratory motion, lung MRI offers additional functional imaging capacities beyond the scope of X-ray and CT. With this, lung MRI offers not only solutions for tricky problems of daily routine but also is a good option for paediatrics and science or any situation where CT would be (relatively) contra-indicated. A set of dedicated protocols, as suggested in this article, may help new users to get started and to stimulate the further development of this powerful, third modality for lung imaging besides X-ray and CT. Further details on how to use the suggested "toolbox" or "sequence buffet" may be used and modified for specific clinical applications and how future perspectives for further protocol developments would look like will be discussed in the subsequent article of this series.

Acknowledgements The following members of the AG Thorax of the German Roentgen Society (DRG) have approved the protocol suggestions and support the publication as current state of the art:

Das M, Dettmer S, Eibel R, Eichinger M, Fink C, Frank J, Hamer O, Hoffmann-Preiß K, Kauczor HU, Kirchner J, Langer M, Ley S, Lörcher U, Ludwig K, Nikolaou K, Pauls S, Poschenrieder F, Rehbock B, Reuter M, Rossdeutscher R, Schaefer JF, Vogel-Claussen J, Diederich S, Tuengerthal S, Plathow C, Poschenrieder F. This initiative was supported by a grant of the German Research Foundation (DFG)

\section{References}

1. Puderbach M, Hintze C, Ley S, Eichinger M, Kauczor H-U, Biederer J (2007) MR imaging of the chest: a practical approach at $1.5 \mathrm{~T}$. Eur J Radiol 64:345-55

2. Eibel R, Herzog P, Dietrich O, Rieger CT, Ostermann H, Reiser MF, Schoenberg SO (2006) Pulmonary abnormalities in immunocompromised patients: comparative detection with parallel acquisition MR imaging and thin-section helical CT. Radiology 241:880-891

3. Biederer J (2005) Magnetic resonance imaging: technical aspects and recent developments. Med Klin (Munich) 100:62-72

4. Lotz J, Kivelitz D, Fischbach R, Beer M, Miller S (2009) Recommendations for utilizing computerized tomography and magnetic resonance tomography in heart diagnosis. 2-Magnetic resonance tomography. Rofo 181:800-814

5. Biederer J, Bauman G, Hintze C, Fabel M, Both M (2011) Magnetresonanztomographie. Der Pneumologe 8:234-242

6. Bankier AA, O'Donnell CR, Mai VM, Storey P, De Maertelaer V, Edelman RR, Chen Q (2004) Impact of lung volume on MR signal intensity changes of the lung parenchyma. J Magn Reson Imaging 20:961-966

7. Bauman G, Puderbach M, Deimling M, Jellus V, Chefd'hotel C, Dinkel J, Hintze C, Kauczor H-U, Schad LR (2009) Non-contrastenhanced perfusion and ventilation assessment of the human lung by means of fourier decomposition in proton MRI. Magn Reson Med 62:656-664

8. Biederer J, Hintze C, Fabel M (2008) MRI of pulmonary nodules: technique and diagnostic value. Cancer Imaging 8:125-130

9. Iwasawa T, Takahashi H, Ogura T, Asakura A, Gotoh T, Kagei S, J-ichi N, Obara M, Inoue T (2007) Correlation of lung parenchymal 
MR signal intensity with pulmonary function tests and quantitative computed tomography (CT) evaluation: a pilot study. J Magn Reson Imaging 26:1530-1536

10. Bauman G, Lützen U, Ullrich M, Gaass T, Dinkel J, Elke G, Meybohm P, Frerichs I, Hoffmann B et al (2011) Pulmonary functional imaging: qualitative comparison of Fourier decomposition MR imaging with SPECT/CT in porcine lung. Radiology 260:551-559

11. Ley-Zaporozhan J, Ley S, Eberhardt R, Kauczor H-U, Heussel CP (2010) Visualization of morphological parenchymal changes in emphysema: comparison of different MRI sequences to $3 \mathrm{D}$ HRCT. Eur J Radiol 73:43-49

12. Biederer J, Busse I, Grimm J, Reuter M, Muhle C, Freitag S, Heller M (2002) Sensitivity of MRI in detecting alveolar Infiltrates: Experimental studies. Rofo 174:1033-9

13. Kersjes W, Hildebrandt G, Cagil H, Schunk K, von Zitzewitz H, Schild H (1999) Differentiation of alveolitis and pulmonary fibrosis in rabbits with magnetic resonance imaging after intrabronchial administration of bleomycin. Invest Radiol 34:13-21

14. Fink C, Puderbach M, Biederer J, Fabel M, Dietrich O, Kauczor H-U, Reiser MF, Schönberg SO (2007) Lung MRI at 1.5 and 3 Tesla: observer preference study and lesion contrast using five different pulse sequences. Invest Radiol 42:377-83

15. Jacob RE, Amidan BG, Soelberg J, Minard KR (2010) In vivo MRI of altered proton signal intensity and T2 relaxation in a bleomycin model of pulmonary inflammation and fibrosis. J Magn Reson Imaging 31:1091-1099

16. Rieger C, Herzog P, Eibel R, Fiegl M, Ostermann H (2008) Pulmonary MRI-a new approach for the evaluation of febrile neutropenic patients with malignancies. Support Care Cancer 16:599-606

17. Fink C, Puderbach M, Biederer J, Fabel M, Dietrich O, Kauczor H-U, Reiser MF, Schönberg SO (2007) Lung MRI at 1.5 and 3 Tesla: observer preference study and lesion contrast using five different pulse sequences. Invest Radiol 42:377-83

18. Biederer J, Schoene A, Freitag S, Reuter M, Heller M (2003) Simulated pulmonary nodules implanted in a dedicated porcine chest phantom: sensitivity of MR imaging for detection. Radiology 227:475-83

19. Both M, Schultze J, Reuter M, Bewig B, Hubner R, Bobis I, Noth R, Heller M, Biederer J (2005) Fast T1- and T2-weighted pulmonary MR-imaging in patients with bronchial carcinoma. Eur J Radiol 53:478-88

20. Bruegel M, Gaa J, Woertler K, Ganter C, Waldt S, Hillerer C, Rummeny EJ (2007) MRI of the lung: value of different turbo spin-echo, single-shot turbo spin-echo, and 3D gradient-echo pulse sequences for the detection of pulmonary metastases. J Magn Reson Imaging 25:73-81

21. Fabel M, Wintersperger BJ, Dietrich O, Eichinger M, Fink C, Puderbach M, Kauczor H-U, Schoenberg SO, Biederer J (2009) MRI of respiratory dynamics with 2D steady-state free-precession and 2D gradient echo sequences at 1.5 and 3 Tesla: an observer preference study. Eur Radiol 19:391-9

22. Gamsu G, de Geer G, Cann C, Müller N, Brito A (1987) A preliminary study of MRI quantification of simulated calcified pulmonary nodules. Invest Radiol 22:853-858

23. Regier M, Kandel S, Kaul MG, Hoffmann B, Ittrich H, Bansmann PM, Kemper J, Nolte-Ernsting C, Heller M et al (2007) Detection of small pulmonary nodules in high-field MR at $3 \mathrm{~T}$ : evaluation of different pulse sequences using porcine lung explants. Eur Radiol $17: 1341-51$

24. Chung MH, Lee HG, Kwon SS, Park SH (2000) MR imaging of solitary pulmonary lesion: emphasis on tuberculomas and comparison with tumors. J Magn Reson Imaging 11:629-637

25. Kersjes W, Mayer E, Buchenroth M, Schunk K, Fouda N, Cagil H (1997) Diagnosis of pulmonary metastases with turbo-SE MR imaging. Eur Radiol 7:1190-1194
26. Kirchner J, Kirchner EM (2001) Melanoptysis: findings on CT and MRI. Br J Radiol 74:1003-1006

27. Baumann T, Ludwig U, Pache G, Gall C, Saueressig U, Fisch D, Stankovic Z, Bartholomae J-P, Honal Mua (2008) Detection of pulmonary nodules with move-during-scan magnetic resonance imaging using a free-breathing turbo inversion recovery magnitude sequence. Invest Radiol 43:359-367

28. Khalil AM, Carette MF, Cadranel JL, Mayaud CM, Akoun GM, Bigot JM (1994) Magnetic resonance imaging findings in pulmonary Kaposi's sarcoma: a series of 10 cases. Eur Respir J 7:12851289

29. Semelka RC, Cem Balci N, Wilber KP, Fisher LL, Brown MA, Gomez-Caminero A, Molina PL (2000) Breath-hold 3D gradientecho MR imaging of the lung parenchyma: evaluation of reproducibility of image quality in normals and preliminary observations in patients with disease. J Magn Reson Imaging 11:195-200

30. Biederer J, Both M, Graessner J, Liess C, Jakob P, Reuter M, Heller M (2003) Lung morphology: fast MR imaging assessment with a volumetric interpolated breath-hold technique: initial experience with patients. Radiology 226:242-9

31. Chen W, Jian W, H-tao L, Li C, Y-ke Z, Xie B, D-quan Z, Y-ming D, Lin Yua (2010) Whole-body diffusion-weighted imaging vs. FDG-PET for the detection of non-small-cell lung cancer. How do they measure up? Magn Reson Imaging 28:613-620

32. Yi CA, Shin KM, Lee KS, Kim B-T, Kim H, Kwon OJ, Choi JY, Chung MJ (2008) Non-small cell lung cancer staging: efficacy comparison of integrated PET/CT versus 3.0-T whole-body MR imaging. Radiology 248:632-642

33. Hasegawa I, Boiselle PM, Kuwabara K, Sawafuji M, Sugiura H (2008) Mediastinal lymph nodes in patients with non-small cell lung cancer: preliminary experience with diffusion-weighted MR imaging. J Thorac Imaging 23:157-161

34. Pauls S, Schmidt SA, Juchems MS, Klass O, Luster M, Reske SN, Brambs H-J, Feuerlein S (2012) Diffusion-weighted MR imaging in comparison to integrated [(18)F]-FDG PET/CT for N-staging in patients with lung cancer. Eur J Radiol 81:178-182

35. Koyama H, Ohno Y, Aoyama N, Onishi Y, Matsumoto K, Nogami M, Takenaka D, Nishio W, Ohbayashi C et al (2010) Comparison of STIR turbo SE imaging and diffusion-weighted imaging of the lung: capability for detection and subtype classification of pulmonary adenocarcinomas. Eur Radiol 20:790-800

36. Liu H, Liu Y, Yu T, Ye N (2010) Usefulness of diffusion-weighted MR imaging in the evaluation of pulmonary lesions. Eur Radiol 20:807-815

37. Tondo F, Saponaro A, Stecco A, Lombardi M, Casadio C, Carriero A (2011) Role of diffusion-weighted imaging in the differential diagnosis of benign and malignant lesions of the chest-mediastinum. Radiol Med 116:720-733

38. Kanauchi N, Oizumi H, Honma T, Kato H, Endo M, Suzuki J, Fukaya K, Sadahiro M (2009) Role of diffusion-weighted magnetic resonance imaging for predicting of tumor invasiveness for clinical stage IA non-small cell lung cancer. Eur J Cardiothorac Surg 35:706710; discussion 710-711

39. Qi LP, Zhang XP, Tang L, Li J, Sun YS, Zhu GY (2009) Using diffusion-weighted MR imaging for tumor detection in the collapsed lung: a preliminary study. Eur Radiol 19:333-341

40. Uto T, Takehara Y, Nakamura Y, Naito T, Hashimoto D, Inui N, Suda T, Nakamura H, Chida K (2009) Higher sensitivity and specificity for diffusion-weighted imaging of malignant lung lesions without apparent diffusion coefficient quantification. Radiology 252:247-254

41. Karabulut N (2009) Accuracy of diffusion-weighted MR imaging for differentiation of pulmonary lesions. Radiology 253:899; author reply 899-900

42. Henzler T, Schmid-Bindert G, Schoenberg SO, Fink C (2010) Diffusion and perfusion MRI of the lung and mediastinum. Eur $\mathrm{J}$ Radiol 76:329-336 
43. Regier M, Schwarz D, Henes FO, Groth M, Kooijman H, Begemann PG, Adam G (2011) Diffusion-weighted MR-imaging for the detection of pulmonary nodules at 1.5 Tesla: intraindividual comparison with multidetector computed tomography. J Med Imaging Radiat Oncol 55:266-274

44. Biederer J, Hintze C, Fabel M (2008) MRI of pulmonary nodules: technique and diagnostic value. Cancer Imaging 8:125-30

45. Kluge A, Müller C, Hansel J, Gerriets T, Bachmann G (2004) Real-time MR with TrueFISP for the detection of acute pulmonary embolism: initial clinical experience. Eur Radiol 14:709-718

46. Kluge A, Gerriets T, Stolz E, Dill T, Mueller K-D, Mueller C, Bachmann G (2006) Pulmonary perfusion in acute pulmonary embolism: agreement of MRI and SPECT for lobar, segmental and subsegmental perfusion defects. Acta Radiol 47:933-940

47. Kluge A, Gerriets T, Müller C, Ekinci O, Neumann T, Dill T, Bachmann G (2005) Thoracic real-time MRI: experience from 2200 examinations in acute and ill-defined thoracic diseases. Rofo 177:1513-1521

48. Moody AR, Liddicoat A, Krarup K (1997) Magnetic resonance pulmonary angiography and direct imaging of embolus for the detection of pulmonary emboli. Invest Radiol 32:431-440

49. Moody AR (2003) Magnetic resonance direct thrombus imaging. J Thromb Haemost 1:1403-1409

50. Biederer J, Liess C, Charalambous N, Heller M (2004) Volumetric interpolated contrast-enhanced MRA for the diagnosis of pulmonary embolism in an ex vivo system. J Magn Reson Imaging 19:428-37

51. Matsuoka S, Uchiyama K, Shima H, Terakoshi H, Oishi S, Nojiri Y, Ogata H (2002) Effect of the rate of gadolinium injection on magnetic resonance pulmonary perfusion imaging. J Magn Reson Imaging 15:108-113

52. Meaney JF, Weg JG, Chenevert TL, Stafford-Johnson D, Hamilton BH, Prince MR (1997) Diagnosis of pulmonary embolism with magnetic resonance angiography. N Engl J Med 336:1422-1427

53. Gupta A, Frazer CK, Ferguson JM, Kumar AB, Davis SJ, Fallon MJ, Morris IT, Drury PJ, Cala LA (1999) Acute pulmonary embolism: diagnosis with MR angiography. Radiology 210:353-359

54. Oudkerk M, van Beek EJR, Wielopolski P, van Ooijen PMA, Brouwers-Kuyper EMJ, Bongaerts AHH, Berghout A (2002) Comparison of contrast-enhanced magnetic resonance angiography and conventional pulmonary angiography for the diagnosis of pulmonary embolism: a prospective study. Lancet 359:1643-1647

55. Goyen M, Laub G, Ladd ME, Debatin JF, Barkhausen J, Truemmler KH, Bosk S, Ruehm SG (2001) Dynamic 3D MR angiography of the pulmonary arteries in under four seconds. J Magn Reson Imaging 13:372-377

56. Stein PD, Chenevert TL, Fowler SE, Goodman LR, Gottschalk A, Hales CA, Hull RD, Jablonski KA, Leeper KV et al (2010) Gadolinium-enhanced magnetic resonance angiography for pulmonary embolism: a multicenter prospective study (PIOPED III). Ann Intern Med 152:434-443, W142-W143

57. Eichinger M, Puderbach M, Fink C, Gahr J, Ley S, Plathow C, Tuengerthal S, Zuna I, Müller F-M et al (2006) Contrast-enhanced 3D MRI of lung perfusion in children with cystic fibrosis-initial results. Eur Radiol 16:2147-52

58. Ersoy H, Goldhaber SZ, Cai T, Luu T, Rosebrook J, Mulkern R, Rybicki F (2007) Time-resolved MR angiography: a primary screening examination of patients with suspected pulmonary embolism and contraindications to administration of iodinated contrast material. AJR Am J Roentgenol 188:1246-1254

59. Levin DL, Chen Q, Zhang M, Edelman RR, Hatabu H (2001) Evaluation of regional pulmonary perfusion using ultrafast magnetic resonance imaging. Magn Reson Med 46:166-171

60. Arai TJ, Henderson AC, Dubowitz DJ, Levin DL, Friedman PJ, Buxton RB, Prisk GK, Hopkins SR (2009) Hypoxic pulmonary vasoconstriction does not contribute to pulmonary blood flow heterogeneity in normoxia in normal supine humans. J Appl Physiol 106:1057-1064

61. Burnham KJ, Arai TJ, Dubowitz DJ, Henderson AC, Holverda S, Buxton RB, Prisk GK, Hopkins SR (2009) Pulmonary perfusion heterogeneity is increased by sustained, heavy exercise in humans. J Appl Physiol 107:1559-1568

62. Eichinger M, Puderbach M, Fink C, Gahr J, Ley S, Plathow C, Tuengerthal S, Zuna I, Müller F-M et al (2006) Contrast-enhanced 3D MRI of lung perfusion in children with cystic fibrosis-initial results. Eur Radiol 16:2147-52

63. Altes TA, Eichinger M, Puderbach M (2007) Magnetic resonance imaging of the lung in cystic fibrosis. Proc Am Thorac Soc 4:321327

64. Eichinger M, Optazaite D-E, Kopp-Schneider A, Hintze C, Biederer J, Niemann A, Mall MA, Wielpütz MO, Kauczor H-U et al (2011) Morphologic and functional scoring of cystic fibrosis lung disease using MRI. Eur J Radiol (in press)

65. Puderbach M, Eichinger M, Haeselbarth J, Ley S, Kopp-Schneider A, Tuengerthal S, Schmaehl A, Fink C, Plathow C et al (2007) Assessment of morphological MRI for pulmonary changes in cystic fibrosis (CF) patients: comparison to thin-section $\mathrm{CT}$ and chest x-ray. Invest Radiol 42:715-725

66. Puderbach $\mathrm{M}$, Eichinger $\mathrm{M}$, Gahr J, Ley S, Tuengerthal S, Schmähl A, Fink C, Plathow C, Wiebel M et al (2007) Proton MRI appearance of cystic fibrosis: comparison to CT. Eur Radiol 17:716-724

67. Biederer J, Reuter M, Both M, Muhle C, Grimm J, Graessner J, Heller M (2002) Analysis of artefacts and detail resolution of lung MRI with breath-hold T1-weighted gradient-echo and T2weighted fast spin-echo sequences with respiratory triggering. Eur Radiol 12:378-384

68. Fabel M, Wintersperger BJ, Dietrich O, Eichinger M, Fink C, Puderbach M, Kauczor H-U, Schoenberg SO, Biederer J (2009) MRI of respiratory dynamics with $2 \mathrm{D}$ steady-state free-precession and 2D gradient echo sequences at 1.5 and 3 Tesla: an observer preference study. Eur Radiol 19:391-9

69. Biederer J, Dinkel J, Bolte H, Welzel T, Hoffmann B, Thierfelder C, Mende U, Debus J, Heller M et al (2007) Respiratory-gated helical computed tomography of lung: reproducibility of small volumes in an ex vivo model. Int J Radiat Oncol Biol Phys 69:1642-9

70. Cai J, Read PW, Altes TA, Molloy JA, Brookeman JR, Sheng K (2007) Evaluation of the reproducibility of lung motion probability distribution function (PDF) using dynamic MRI. Phys Med Biol $52: 365-373$

71. Adamson J, Chang Z, Wang Z, Yin F-F, Cai J (2010) Maximum intensity projection (MIP) imaging using slice-stacking MRI. Med Phys 37:5914-5920

72. Tetzlaff R, Schwarz T, Kauczor H-U, Meinzer H-P, Puderbach M, Eichinger M (2010) Lung function measurement of single lungs by lung area segmentation on 2D dynamic MRI. Acad Radiol 17:496503

73. Wild JM, Schmiedeskamp J, Paley MNJ, Filbir F, Fichele S, Kasuboski L, Knitz F, Woodhouse N, Swift A et al (2002) MR imaging of the lungs with hyperpolarized helium-3 gas transported by air. Phys Med Biol 47:N185-N190

74. Scholz A-W, Wolf U, Fabel M, Weiler N, Heussel CP, Eberle B, David M, Schreiber WG (2009) Comparison of magnetic resonance imaging of inhaled SF6 with respiratory gas analysis. Magn Reson Imaging 27:549-556

75. Molinari F, Puderbach M, Eichinger M, Ley S, Fink C, Bonomo L, Kauczor H-U, Bock M (2008) Oxygen-enhanced magnetic resonance imaging: influence of different gas delivery methods on the T1-changes of the lungs. Invest Radiol 43:427-432

76. Suga K, Ogasawara N, Tsukuda T, Matsunaga N (2002) Assessment of regional lung ventilation in dog lungs with Gd-DTPA aerosol ventilation MR imaging. Acta Radiol 43:282-291 
77. Attenberger UI, Ingrisch M, Dietrich O, Herrmann K, Nikolaou K, Reiser MF, Schönberg SO, Fink C (2009) Time-resolved 3D pulmonary perfusion MRI: comparison of different $\mathrm{k}$-space acquisition strategies at 1.5 and $3 \mathrm{~T}$. Invest Radiol 44:525-531

78. Peltola V, Ruuskanen O, Svedström E (2008) Magnetic resonance imaging of lung infections in children. Pediatr Radiol 38:12251231

79. Ley-Zaporozhan J, Ley S, Sommerburg O, Komm N, Müller F-MC, Schenk JP (2009) Clinical application of MRI in children for the assessment of pulmonary diseases. Rofo 181:419-432

80. Failo R, Wielopolski PA, Tiddens HAWM, Hop WCJ, Mucelli RP, Lequin MH (2009) Lung morphology assessment using MRI: a robust ultra-short TR/TE 2D steady state free precession sequence used in cystic fibrosis patients. Magn Reson Med 61:299 306

81. Wagner M, Böwing B, Kuth R, Deimling M, Rascher W, Rupprecht T (2001) Low field thoracic MRI-a fast and radiation free routine imaging modality in children. Magn Reson Imaging 19:975-983

82. Rupprecht T, Kuth R, Bowing B, Gerling S, Wagner M, Rascher W (2000) Sedation and monitoring of paediatric patients undergoing open low-field MRI. Acta Paediatr 89:1077-1081

83. Serra G, Milito C, Mitrevski M, Granata G, Martini H, Pesce AM, Sfika I, Bonanni L, Catalano C et al (2011) Lung MRI as a possible alternative to $\mathrm{CT}$ scan for patients with primary immune deficiencies and increased radio sensitivity. Chest 140:1581-1589

84. Hirsch W, Sorge I, Krohmer S, Weber D, Meier K, Till H (2008) MRI of the lungs in children. Eur J Radiol 68:278-288

85. Schaefer JF, Kramer U (2011) Whole-body MRI in children and juveniles. Rofo 183:24-36
86. Sanborn PA, Michna E, Zurakowski D, Burrows PE, Fontaine PJ, Connor L, Mason KP (2005) Adverse cardiovascular and respiratory events during sedation of pediatric patients for imaging examinations. Radiology 237:288-294

87. Lutterbey G, Wattjes MP, Doerr D, Fischer NJ, Gieseke J Jr, Schild $\mathrm{HH}$ (2007) Atelectasis in children undergoing either propofol infusion or positive pressure ventilation anesthesia for magnetic resonance imaging. Paediatr Anaesth 17:121-125

88. Biederer J (2009) General requirements of MRI of the lung and suggested standard protocol. In: Kauczor H-U (ed) MRI of the lung. Springer, Berlin Heidelberg, pp 3-16

89. Biederer J, Puderbach M (2009) MR imaging of the chest. HIRE $3: 39-42$

90. Puderbach M, Hintze C, Ley S, Eichinger M, Kauczor H-U, Biederer J (2007) MR imaging of the chest: a practical approach at 1.5 T. Eur J Radiol 64:345-55

91. Hintze C, Biederer J, Kauczor H-U (2007) Magnetic resonance imaging of the chest. In: Magnevist Monograph. Springer, Berlin, Heidelberg, New York, pp 87-103

92. BÄK Bundesärztekammer (2006) Bundesärztekammer-MRT Qualitätssicherung. http://www.bundesaerztekammer.de/downloads/MagnetResonanz.pdf

93. Stein PD, Gottschalk A, Sostman HD, Chenevert TL, Fowler SE, Goodman LR, Hales CA, Hull RD, Kanal E et al (2008) Methods of prospective investigation of pulmonary embolism diagnosis III (PIOPED III). Semin Nucl Med 38:462-470

94. Kluge A, Luboldt W, Bachmann G (2006) Acute pulmonary embolism to the subsegmental level: diagnostic accuracy of three MRI techniques compared with 16-MDCT. AJR Am J Roentgenol 187:W7-W14 